\title{
INTRODUCCIÓN DEL GEOGEBRA EN EL PROCESO DE ENSEÑANZA-APRENDIZAJE DE GEOMETRÍA A DOCENTES EN FORMACIÓN
}

\section{Introduction of Geogebra in the Teaching-Learning \\ Process of Geometry to Teachers in Training}

Rogel Rafael Rojas-Bello ${ }^{1}$

rogel.rojas@isfodosu.edu.do

${ }^{1}$ Instituto Superior de Formación Docente Salomé Ureña, República Dominicana

Fecha recepción: 24/10/2019

Fecha aprobación: 02/1/2020

\section{Resumen}

En este trabajo se presenta una experiencia didáctica de la mediación del programa informático GeoGebra en el proceso de enseńanza-aprendizaje de la asignatura Geometría en estudiantes de la licenciatura en Matemática en una institución superior de formación de docentes. La metodología consiste en clases cooperativas entre docente y estudiantes, y son apoyadas en una diversidad de situaciones problemáticas por el software GeoGebra, el cual posee un compendio de elementos que permiten plantear diferentes experiencias de enseñanza-aprendizaje, es de fácil manejo y posee representaciones gráficas robustas. Se describen diversas experiencias que se llevaron a cabo en el aula, evidenciándose una buena participación y motivación de los estudiantes en el trabajo de aula individual y colaborativo, además de la apropiación por parte de los estudiantes de conocimientos geométricos tanto en coordenadas cartesianas de dos y tres dimensiones, como en coordenadas polares.

Palabras claves: formación de docentes, GeoGebra, Geometría, simuladores, tecnología educativa, recurso educativo.

\section{Abstract}

This paper presents a didactic experience of the mediation of the GeoGebra software in the teaching-learning process of the Geometry III course in students of the Bachelor's degree in Mathematics in a teacher training institution. The methodology consists of cooperative classes between teachers and students, and they are supported in a variety of problematic situations by the GeoGebra software which has a compendium of elements that allow different teaching-learning experiences, is easy to use and has robust graphic representations. Several experiences that were conducted in the classroom are described, showing a good participation and motivation of the students in the individual and collaborative classroom work, in addition to the appropriation by the students of geometric knowledge in two- and three-dimensional Cartesian coordinates, as in polar coordinates.

Keywords: educational technology, Geometry, GeoGebra, mathematics software, teacher education. 


\section{Introducción}

En el desafío de construir una sociedad más justa y equitativa a partir de una educación de calidad, democrática e incluyente, debe aparecer entre las principales líneas de acción en la educación científica y tecnológica de cualquier país. Para la formación de ciudadanos reflexivos se requiere la adquisición de códigos científicos básicos, necesarios para participar en las decisiones que se deben tomar para definir el ritmo y los propósitos de los cambios. Hoy en día, el avance tecnológico ha hecho posible el surgimiento de una serie de herramientas de cómputo, que realizan operaciones repetitivas y cálculos engorrosos, entre los que podemos mencionar los lenguajes Java, $\mathrm{C}++$ y Python, entre otros.

Por otra parte, son de particular importancia por su fácil manejo y las salidas gráficas y robustas, los denominados manipuladores o softwares algebraicos y geométricos, que apoyan enormemente el proceso de enseñanza-aprendizaje de las Matemáticas, entre estos se puede mencionar el GeoGebra (2019), el cual posee un compendio de elementos que permiten plantear diferentes experiencias de aprendizaje. Este software es de fácil acceso y su uso se ha ido extendiendo a la enseñanza de la Matemáticas y en particular a la Geometría.

Es una preocupación constante por parte del docente de Matemáticas que sus estudiantes logren un aprendizaje significativo, y para ello es necesario usar todas las herramientas que tenga a su disposición, sobre todo si son estudiantes que serán futuros formadores, pues no solo tienen que aprender Matemáticas, sino, además, aprender a enseñar Matemáticas.

En este trabajo, se describe una experiencia del proceso de enseñanza-aprendizaje que se llevó a cabo en el curso de Geometría, dirigido a maestros en formación, en la licenciatura en Matemática en una institución superior de formación de docentes. Se aplica una metodología colaborativa entre estudiantes y docentes, la cual es apoyada en diversos momentos didácticos por el software GeoGebra.

\section{Revisión de la literatura}

En los últimos años, se han diseñado manipuladores algebraicos que ayudan enormemente en la solución de modelos matemáticos complejos, brindándole al investigador la posibilidad de experimentar situaciones ideales y aplicar el método de ensayo-error en corto tiempo, además de las ventajas de graficar de manera rápida las situaciones del fenómeno objeto de estudio. Al respecto, Socas (2011) afirma que:

El potencial del ordenador para crear ambientes de aprendizaje que difícilmente podrían ser logrados sin disponer de este recurso está fuera de duda. Estos ambientes computacionales requieren, unas veces, la elaboración de programas o códigos para establecer secuencias que permiten desarrollar aspectos operacionales del conocimiento algebraico, así como hacer predicciones. Otras, estos ambientes se centran en las relaciones entre distintas representaciones de objetos matemáticos, poniendo énfasis en los aspectos estructurales, y a veces combinan ambos (p.23).

En este sentido, Godino et al. (2003), citado por Ramírez (2015), realizó una amplia indagación de estudios relacionados con el campo, encontrando que los estudiantes pueden aprender más Matemáticas y de manera más profunda con el uso de una tecnología apropiada sin llegar a cometer el error de usarla como sustituto de intuiciones y comprensiones conceptuales. Además, aconsejan que los recursos tecnológicos se deben usar de manera amplia y responsable con el fin de enriquecer el aprendizaje matemático de los estudiantes.

Por su parte, la Organización de Estados Iberoamericanos (2010, p.115), en su documento final de las Metas Educativas 2021, sobre la incorporación de las TIC, plantea que, para que puedan aprovecharse en su máximo potencial educativo, la mismas han de ser acompañadas y complementadas, además de con los procesos de actualización y mantenimiento propios del equipamiento, con la capacitación para los docentes. Es preciso, por lo tanto, proporcionar al profesor los recursos profesionales que este necesita 
para realizar su labor de la manera más satisfactoria posible.

En el mercado hay una amplia oferta de este tipo de programas, entre ellos se encuentra el GeoGebra, el cual facilita los procesos de abstracción mostrando la construcción de una relación entre un modelo geométrico y un modelo algebraico de una situación de la vida real, lo que permite encontrar soluciones no solo Matemáticas, sino además visuales, que representan la solución de un determinado problema. Además, es de fácil manejo, por lo que el docente o estudiante puede comenzar el trabajo con este paquete, sin necesidad de consumir mucho tiempo en su aprendizaje. Al respecto, Effandi y Lo (2012), citado por Barahona et al. (2015), en su investigación con estudiantes de Matemáticas a nivel universitario, proponen que:

no solo los estudiantes encontraron aspectos motivacionales de aprendizaje a través de GeoGebra, los docentes también tienen percepciones positivas del uso de la herramienta sugiriendo que debe usarse como una alternativa válida en los procesos de enseñanza y aprendizaje de las Matemáticas. A través de varios experimentos de la integración de GeoGebra se demuestra que brinda posibilidades a los estudiantes en el desarrollo de la intuición a través de la visualización de los procesos matemáticos, permitiendo a los estudiantes explorar una variedad de tipos de funciones a través de conexiones entre las representaciones simbólicas y visuales (pp.122-123).

Por su parte, Carranza (2011), citado por Jiménez y Jiménez $(2017$, p.12) en un estudio documental sobre los diversos proyectos y trabajos que usan el GeoGebra en el aula, revela que este software, en la formación de los profesores de Matemática, favorece la construcción de conocimientos matemáticos significativos, operativos y estructurados, lo que les permite movilizarse fácilmente entre los sistemas de representación simbólicos, numéricos, gráficos y analíticos. Además, que el docente puede apoyarse en el GeoGebra para que el estudiante pierda el miedo a las Matemáticas.
Asimismo, un estudio focalizado en docentes en proceso de capacitación en Geometría, usando GeoGebra, Cotic (2014), pudo verificar que: "el uso de las TIC y el empleo de metodologías adecuadas en el aprendizaje de contenidos de Geometría permite a los docentes que se capacitan, revisar conocimientos y procesos que les resultaban muy difíciles de proponer a sus alumnos" (p.5).

Recientemente, Morales-López (2019), asoció la Geometría, su enseñanza y el uso de tecnología con 65 profesores de Matemáticas en formación, utilizando específicamente el GeoGebra, concluyendo que el docente debe definir actividades que eviten el uso indiscriminado de tecnología, incluir en la planificación la comprensión de los conocimientos requeridos, y que además es claro que, si un profesor de Matemáticas no se cuestiona de manera auténtica la forma en que debe orientar las tareas que involucran la tecnología, el lenguaje que debe ser utilizado y el concepto matemático que está interviniendo, no podrá integrar la tecnología pertinentemente en el aula de esta asignatura.

\section{Método}

\subsection{Contexto de la investigación}

La investigación se desarrolló en el cuatrimestre enero-abril de 2019, entre los estudiantes de Geometría, asignatura del quinto semestre de la carrera de licenciatura en Matemática en una institución superior de formación de docentes. Son estudiantes de ambos sexos y edades promedio de 21 años. Se seleccionó dicha asignatura para realizar esta investigación, esencialmente, porque su contenido es clave para la formación de los estudiantes en la Geometría Analítica, en esta se aborda esencialmente el análisis de rectas y planos, la identificación, gráfica y aplicaciones de las denominadas cónicas y cuádricas, además de las operaciones algebraicas, representaciones y transformaciones de ecuaciones en coordenadas polares.

\subsection{Tipo de investigación}

El enfoque metodológico de la presente investigación se circunscribe a la perspectiva de la investiga- 
ción-acción, la cual es entendida como el estudio de una situación social en la que participan maestros y estudiantes, y pretende tratar de forma simultánea conocimientos y cambios, de manera que se unan la teoría y la práctica en el hecho educativo.

Muñoz et al. (2002), en su trabajo sobre la investigación-acción-reflexión, con educadores en proceso de formación, consideran que el proceso es una espiral y que este tiene cuatro momentos: planificación, actuación, observación y reflexión.

Hay una acción cooperativa entre docente y estudiante, que implica un trabajo en equipo, mediante el cual el estudiante reflexiona, opina y toma conciencia de las causas de las dificultades de la actividad; el profesor orienta y sociabiliza y todos toman decisiones para mejorar la práctica educativa.

Lo importante es en realidad lo que el estudiante descubre, que hace y termina convirtiéndose en la base del proceso de toma de conciencia. Así, el individuo se hace más consciente y comprende mejor el proceso de solución de un problema.

Zeichner (2001), citado en Silva-Peña (2012, p.30), tomando en consideración a diversos autores que argumentan las potencialidades que tiene la investigación-acción para el desarrollo profesional docente, plantea que ella ayuda a los profesores a ser más flexibles y abiertos a las nuevas ideas, los hace más proactivos en lo referente a la autoridad externa, acorta la brecha entre las aspiraciones y las realizaciones de los profesores y ayuda a desarrollar una actitud para el autoanálisis, las cuales se aplican a otras situaciones.

Los instrumentos de investigación son las entrevistas abiertas, la prueba diagnóstica, fotos, notas de campo y evaluaciones docentes, a través de los cuales se recogió la información, esto es, las percepciones y opiniones de estudiantes y docentes, además de datos cuantitativos. Con relación a ello, González (2010) afirma que:

diversos estudios han logrado demostrar que las concepciones sobre el aprendizaje, las formas de abordarlo y las percepciones sobre la situación en que se lleva a cabo tienden a aso- ciarse consistentemente entre ellas y con los resultados obtenidos por los alumnos. Los estudiantes que abordan el aprendizaje de manera profunda presentan mayoritariamente concepciones cualitativas y percepciones positivas sobre el contexto (p.28).

Antes de la ejecución de las actividades, se planificó con antelación las estrategias para la acción pedagógica, en relación con los diferentes contenidos programáticos, y se previeron los recursos para su desarrollo.

Cabe destacar que no todas las actividades en clases incluyen el uso del GeoGebra, pues estas son mucho más valiosas cuando nos permiten hacer cosas que sin el software serían complicadas o poco prácticas, es decir, no tiene sentido alguno utilizar el GeoGebra por el solo hecho de utilizarlas.

\section{Resultados}

\subsection{Fase diagnóstica}

Se aplicó una prueba diagnóstica al inicio de la asignatura Geometría, pretendiendo obtener datos representativos, para entender en qué estado están los conocimientos previos de los estudiantes en términos generales, para así tomar decisiones oportunas con miras a planificar las clases, de tal manera que estas respondan mejor a las necesidades de aprendizaje de los estudiantes.

En la prueba diagnóstica, se les examinó a los estudiantes lo relativo a los conjuntos numéricos, los cuales están relacionados directamente con las magnitudes que se estudian en Geometría, el Teorema de Pitágoras, uno de los principales postulados de la Geometría, la ubicación de puntos, tanto en el plano como en el espacio, además, la representación gráfica de posibles lugares geométricos en el plano.

Se usó una escala de apreciación como instrumento para la obtención de información relevante sobre los conocimientos previos de los estudiantes, que incorpora un gradiente de desempeño, que será expresado en una escala numérica (por ciento). Ello implica observar no solo la presencia o ausencia de una conduc- 
ta en la prueba diagnóstica, sino el grado de intensidad de esta manifestación. En este caso, se usaron cuatro opciones de respuesta: siempre, muchas veces, algunas veces y nunca. Al respecto, con el objeto de brindar apoyo a sus docentes, la Dirección de Calidad Educativa de la Universidad Central de Chile (2017), recomienda que: "El evaluador debe apreciar o estimar la intensidad de dicha conducta o rasgo a lo menos en tres categorías" (p.42).

Los resultados cuantitativos de la prueba diagnóstica que se muestran en la Tabla 1, evidencian que los bajos niveles de logro en los estudiantes se acentúan en las representaciones gráficas, identificación de ecua- ciones y en la localización de puntos en el espacio euclídeo. Esto último fue tratado y se logró consolidar inmediatamente. Por el contrario, cuando se trata de enunciar y representar el Teorema de Pitágoras o de localizar debidamente puntos en el plano, los estudiantes lo hacen adecuadamente, lo cual representa un buen punto de partida. Como recomienda Calvo (2008), es fundamental para la enseñanza significativa de la Matemática, que el docente busque la conexión entre el aprendizaje nuevo con los conocimientos que ya posee el estudiante, debe además aprovecharlos fomentando así la confianza en sí mismo al reconocer que la información que traen consigo es muy importante para el proceso de enseńanza.

Tabla 1. Escala de apreciación de la prueba diagnóstica

\begin{tabular}{|c|c|c|c|c|}
\hline \multirow{2}{*}{ Indicadores } & \multicolumn{4}{|c|}{ Categorías (\%) } \\
\hline & Siempre & Muchas veces & Algunas veces & Nunca \\
\hline $\begin{array}{l}\text { Utiliza correctamente el } \\
\text { signo de } \in 0 \notin \text {. }\end{array}$ & & 33.33 & 66.67 & \\
\hline $\begin{array}{l}\text { Enuncia el Teorema de } \\
\text { Pitágoras correctamente. }\end{array}$ & 66.67 & 33.33 & & \\
\hline $\begin{array}{l}\text { Grafica la situación del } \\
\text { Teorema de Pitágoras. }\end{array}$ & 66.67 & & & 33.33 \\
\hline $\begin{array}{l}\text { Localiza con precisión los } \\
\text { puntos en } \mathbb{R}^{2}\end{array}$ & 83.33 & & 16.67 & \\
\hline $\begin{array}{l}\text { Localiza con precisión los } \\
\text { puntos en } \mathbb{R}^{3} \text {. }\end{array}$ & & & & 100 \\
\hline $\begin{array}{l}\text { Demuestra conocer la defi- } \\
\text { nición de circunferencia. }\end{array}$ & & & 33.33 & 66.67 \\
\hline $\begin{array}{l}\text { Identifica las ecuaciones } \\
\text { en } \mathbb{R}^{3}\end{array}$ & 16.67 & & & 83.33 \\
\hline $\begin{array}{l}\text { Demuestra conocer los } \\
\text { elementos de una ecuación } \\
\text { de segundo grado. }\end{array}$ & & & & 100 \\
\hline
\end{tabular}

Nota: Elaboración propia.

\subsection{Experiencia didáctica}

En esta sección se reporta parte de la experiencia didáctica derivada de las actividades realizadas en el aula. En cada una de las clases, excepto en la primera, los estudiantes están informados con antelación sobre las actividades y contenidos a trabajar. Se comienza con actividades motivacionales, como preguntas de respuestas abiertas y videos que estimulen el asombro de los alumnos, en donde se dan aplicaciones de la Geometría en la Mecánica celeste, Arquitectura, y transmisión-recepción de señales, entre otras; luego se plantean los conceptos y las definiciones, ejemplos, aplicaciones y construcciones de objetos geométricos con asistencia del GeoGebra, cuando la dinámica del tema en el aula de clases lo amerite. Todo en un ambiente colaborativo.

Con miras a abarcar en lo posible lo relativo a rectas y circunferencias: pendiente, perpendicularidad, pa- 
ralelismo, centro, radio, punto medio, distancia entre dos puntos y punto de tangencia; se les propuso a los estudiantes dos situaciones problemáticas, las cuales tienen aplicación cuando se quiere hallar los enlaces en las vistas de una pieza mecánica.

- Problema 1: Hallar las ecuaciones de las rectas tangentes trazadas del punto $(6,-6)$ a la circunferencia de ecuación: $x^{2}+y^{2}+2 x-2 y-23=0$.

- Problema 2: Sea la circunferencia de ecuación: $x^{2}+y^{2}+4 x-10 y-20=0$, hallar las ecuaciones de las rectas tangentes a ella, que son paralelas a la recta de ecuación: $5 x-5 y+31=0$.
En la generalidad de los casos, los estudiantes trazaron un plan y lo pusieron en ejecución paso a paso, hasta lograr, analítica y gráficamente, solucionar los problemas. Además, reflexionan y hacen un resumen de todos los pasos que lo conducen a ese nivel de logro.

En las Figuras 1 y 2 se muestran los gráficos de las situaciones problemáticas 1 y 2, respectivamente, realizados por el estudiante que se llamará E, a quién no le hizo falta recurrir a alguna forma de representación por medio del GeoGebra, pues diseñó y ejecutó un plan, construyendo con lápiz, papel, regla y compás, la situación geométrica, e hizo los cálculos algebraicos necesarios.

\section{Figura 1. Gráfica resultante del problema 1 realizado por el estudiante E}

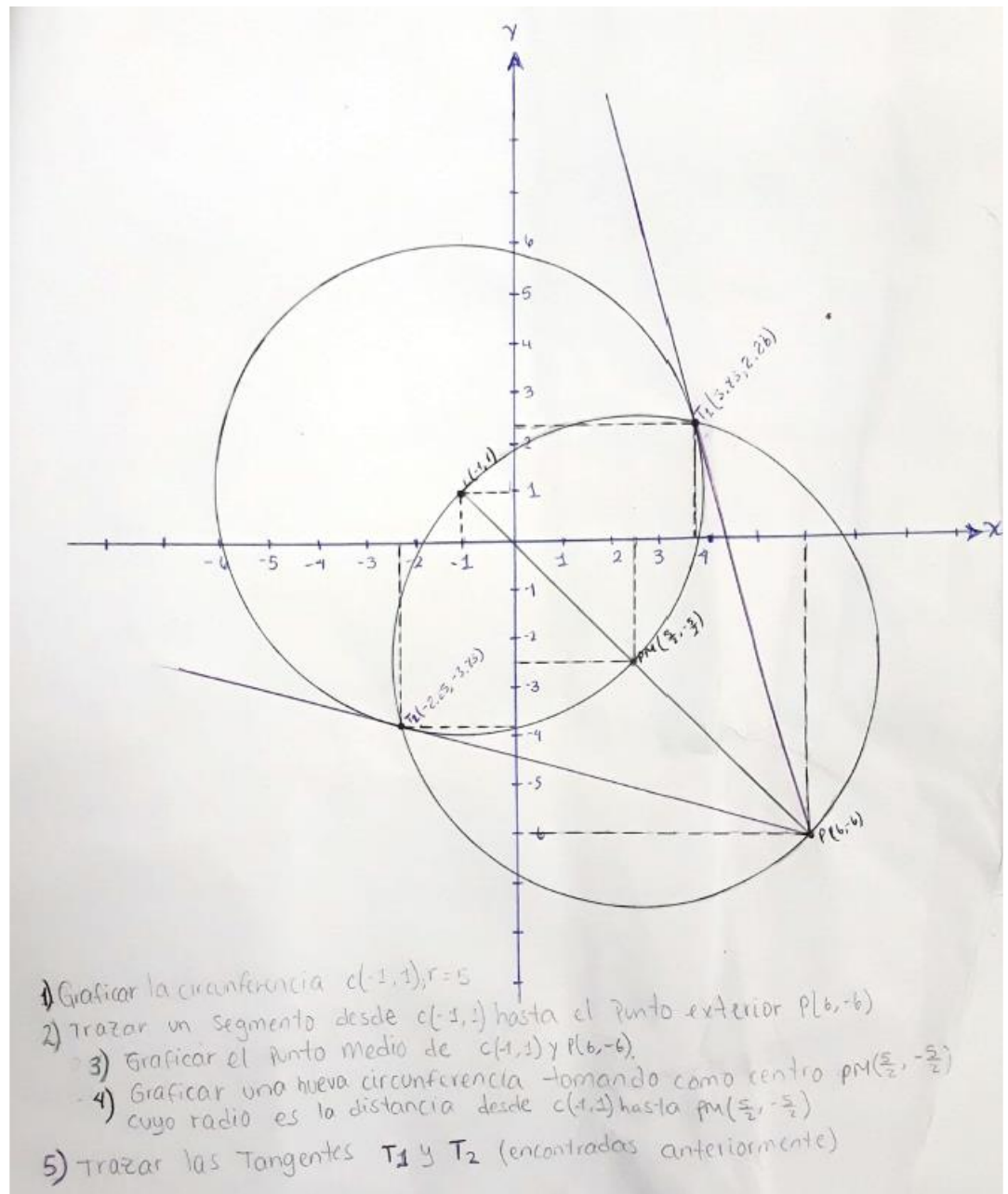

Nota: Elaboración propia. 


\section{Figura 2. Gráfica del problema 2 realizado por el estudiante $\mathrm{E}$}

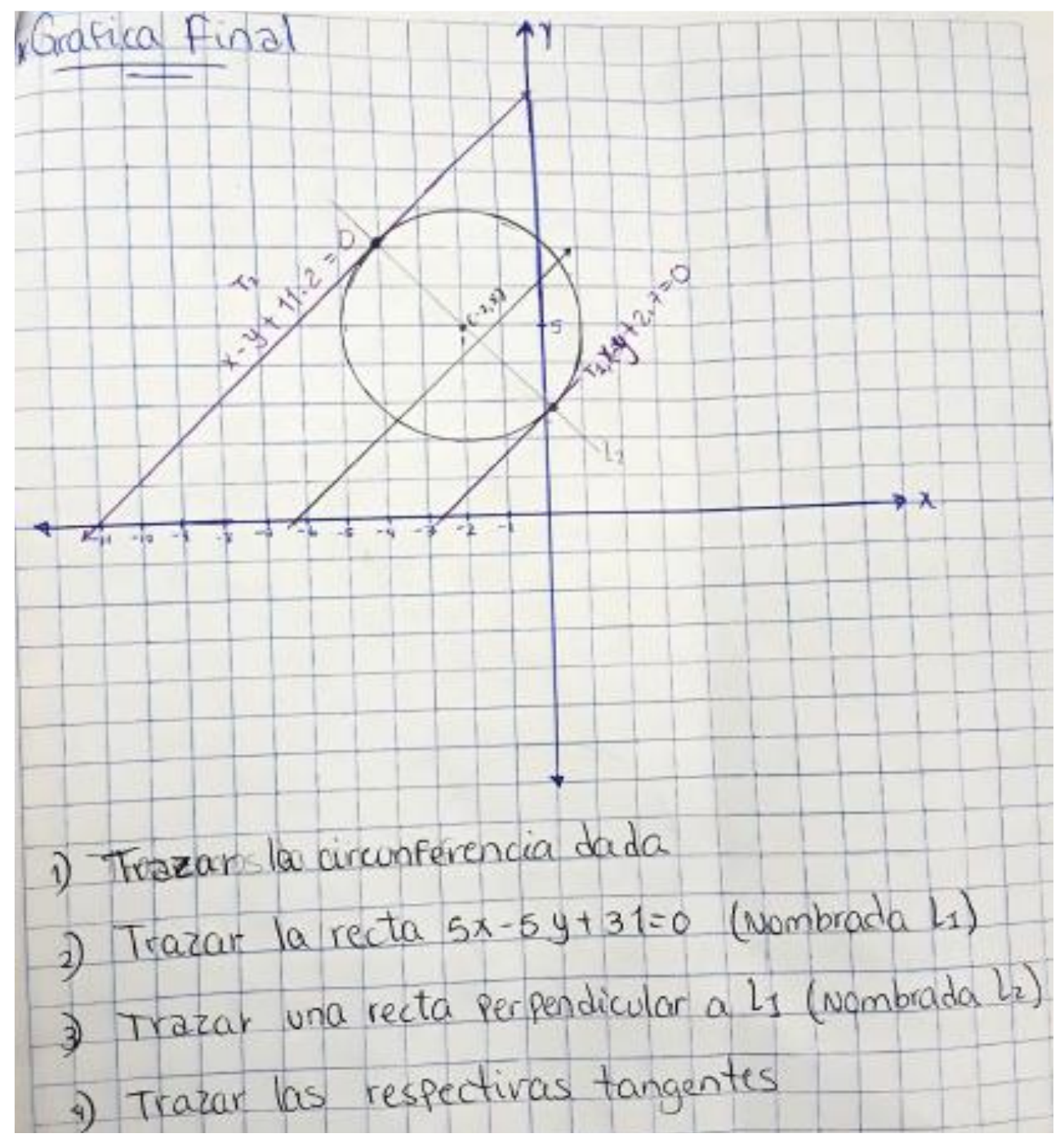

Nota: Elaboración propia.

En el aula, se trabajó lo relativo al sistema de coordenadas polares y las transformaciones a coordenadas cartesianas, y viceversa. La primera gráfica de la ecuación $r=2 \operatorname{Cos}(2 \theta)$ se hizo de manera colaborativa en el pizarrón, asignándole valores a para así obtener valores de $r$ (para este caso $\Theta$ se varió de 0 hasta $\pi)$, luego procedieron a juntar, por medio de líneas, los puntos, de manera secuencial, para así obtener la representación gráfica de la ecuación polar correspondiente, como se muestra en la Figura 3. Por otro lado, en la Figura 4, la misma gráfica, pero con el GeoGebra. Ya que era necesario aumentar el número de puntos para saber con exactitud la representación de la ecuación (hasta $2 \pi$ ), los estudiantes procedieron a hacer la gráfica con el GeoGebra, que resulta una flor de cuatro pétalos (Figura 5).

Con la herramienta disponible, los estudiantes se dieron cuenta que al aumentar el argumento del coseno dos veces, se obtiene otra curva (flor de ocho pétalos), y que hay una relación directa entre el argumento de la ecuación y el número de pétalos de la rosa. Además, se representaron y comentaron, por medio de sus ecuaciones algebraicas, las gráficas más usuales, como Cardiodes, el espiral de Arquímedes y secciones cónicas, entre otras. 
ROJAS-BELLO

Introducción del geogebra en el proceso de enseñanza-aprendizaje de geometría a docentes...

Figura 3. Gráfica aproximada de la curva polar $r=2 \operatorname{Cos}(2 \Theta), \Theta \in[0, \pi]$

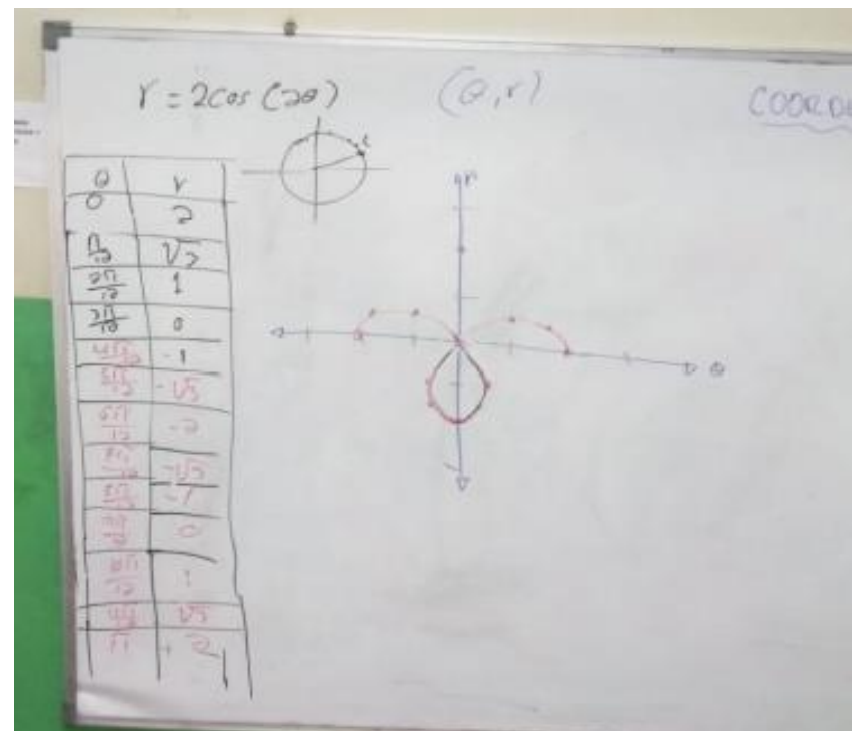

Nota: Elaboración propia.

Figura 4. Gráfica de la curva polar con GeoGebra $r=2 \operatorname{Cos}(2 \theta), \theta \in[0, \pi]$

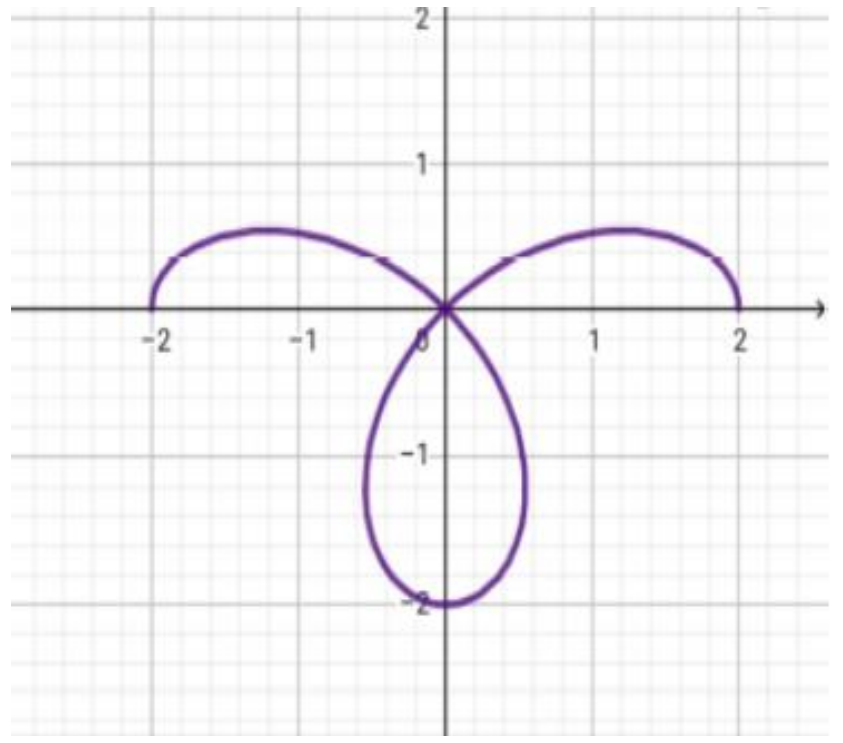

Nota: Elaboración propia.
Figura 5. Gráfica de la curva polar con GeoGebra, $r=2 \operatorname{Cos}(2 \theta), \theta \in[0,2 \pi]$

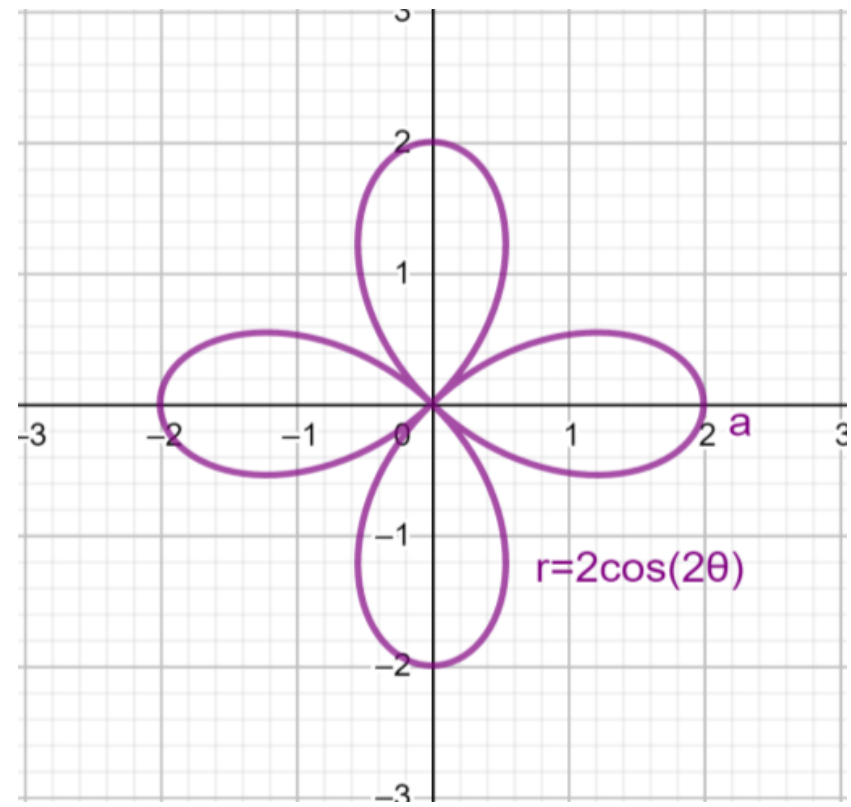

Nota: Elaboración propia.

Figura 6. Gráfica de la curva polar con GeoGebra $r=2 \operatorname{Cos}(4 \Theta), \Theta \in[0,2 \pi]$

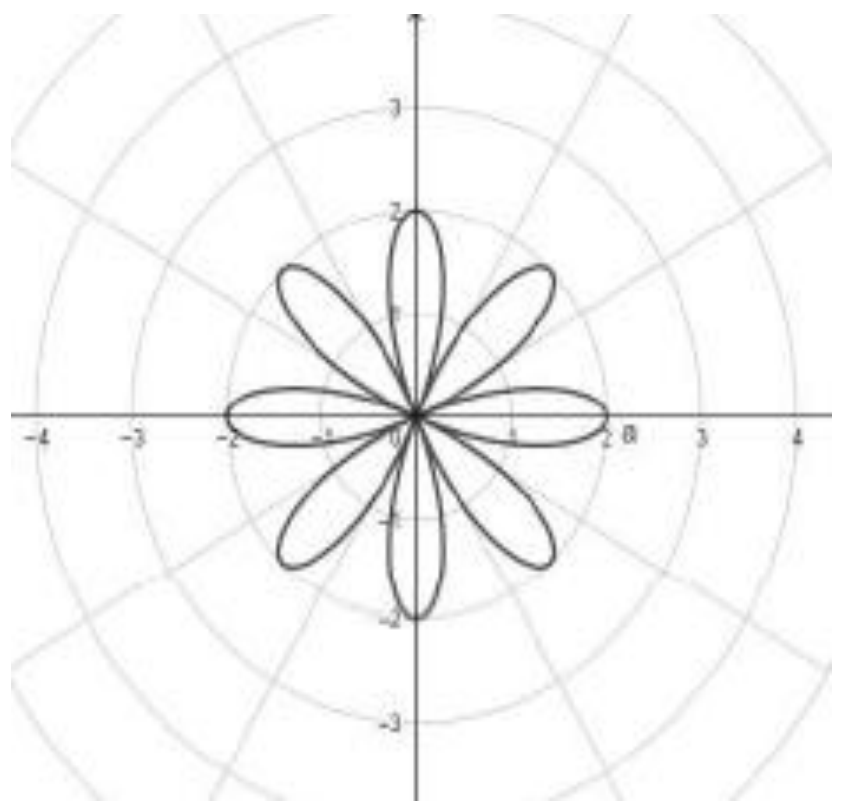

Nota: Elaboración propia. 
Otra experiencia se presentó cuando se estudiaron las cónicas por medio de la ecuación general de segundo grado: $A x^{2}+B y^{2}+C x y+D x+E y+F=0$, con $C=0$. Después de estudiar las diferentes cónicas, con aplicaciones en las leyes que rigen los cuerpos celestes, en Arquitectura y en la transmisión de señales, entre otras, con la presentación de los videos de Luna (2012) y Corella (2012), surgió la inquietud de sustituir a $C$ en la ecuación anterior, con un valor diferente de cero, observándose que no se puede completar cuadrados directamente para hallar el vértice o centro de la posible cónica, entonces procedieron a graficar usando el GeoGebra.

Los estudiantes experimentaron con varias ecuaciones de tal manera que se generaran secciones cónicas con ejes rotados y trasladados, para los casos de la elipse, la hipérbola y la parábola, cuyas ecuaciones algebraicas aparecen en la parte superior derecha, como se muestra en la Figura 7. Es de resaltar que no se puede identificar qué cónica corresponde con solo observar la ecuación, pues es necesario hacer una serie de transformaciones y consideraciones algebraicas.

\section{Figura 7. Secciones cónicas con ejes rotados y trasladados}

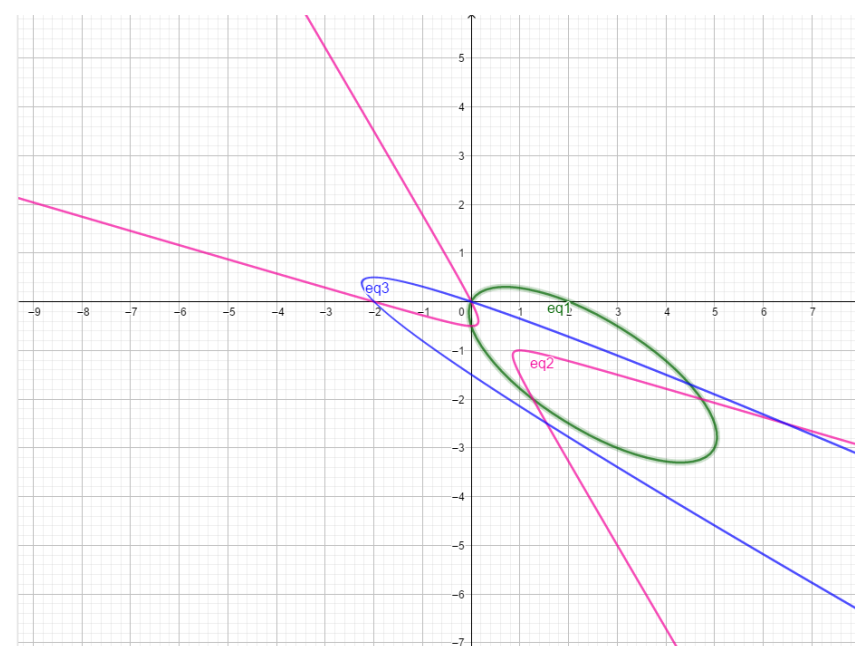

Nota: Elaboración propia.

En el caso del tema de las denominadas cuádricas: esfera, paraboloide, elipsoide, hiperboloide de una y dos hojas y el paraboloide hiperbólico, se realizaron actividades áulicas conducentes a su identificación por medio de ecuaciones algebraicas, cortes con los planos coordenados y gráfica aproximada. En este caso, con el GeoGebra disponible, surgió la curiosidad por parte de los estudiantes de experimentar, generándose con ello múltiples representaciones de todas estas cuádricas, además con la opción que tiene esta herramienta de mirarlas desde distintos ángulos.

\subsection{Opiniones de los estudiantes sobre la meto- dología usada en clase}

Para explorar las percepciones de los estudiantes sobre su aprendizaje, de cara a la metodología usada al término de la asignatura Geometría, les fueron hecha dos preguntas, intentando aglutinar los aspectos más relevantes.

¿Qué opina de la metodología utilizada?

\section{Algunas respuestas:}

- Considero que fue muy satisfactorio, ya que aprendí cada uno de los conceptos tratados. Deberíamos poder tomar más clases con esta metodología.

- Lo que aprendí fue significativo, ya que cuando aprendemos con gráficas (utilizando el GeoGebra) es bueno, explicando los teoremas en conjunto, partiendo de nuestros saberes previos y demás.

- Se ajusta totalmente al modelo por competencias y constructivismo que tanto se persigue en esta nación, el maestro nos enseña Matemáticas y como dar clases.

- Me parece una metodología excelente, ya que en las horas de clase se siente un ambiente de aprendizaje.

- Realizar los ejercicios en la pizarra nos ayuda a comprender mejor todo el procedimiento de forma más detallada, aunque podemos ver videos que nos expliquen el proceso, no es la misma profundidad. 
¿Piensas incluir el uso del GeoGebra en tus futuras clases?

Algunas respuestas:

- Claro que sí, es una herramienta tecnológica que ayuda al estudiante comprender más el área de Geometría.

- Sí, en todo lo que sea de Geometría.

- Es claro que sí, porque creo que, si a mí me funciona para comprender temas, a mis alumnos también les puede funcionar.

- De mi parte, la implementaré como herramienta de trabajo. Es realmente buena.

\section{Conclusiones}

El GeoGebra, usado adecuadamente, permite al futuro maestro obtener mayor profundidad en el estudio de la Geometría, además desarrolla competencias tecnológicas en el proceso de la solución de los problemas propuestos en clase. Por ejemplo, los estudiantes manipulaban los datos iniciales de los problemas para observar el efecto en la simulación, obteniendo obteniendo, con ello, nuevas familias de objetos geométricos, ilustrando mejor algunos conceptos y definiciones; esto es, desarrollan un plan, lo ejecutan y reflexionan sobre la solución obtenida y sus posibles relaciones con situaciones problemáticas similares o nuevas, fortaleciéndose el proceso de reafirmación de lo ya aprendido.

Es necesario el oportuno trabajo en el aula por parte del docente en mediar definiciones, axiomas y teoremas, pues se puede incurrir en el error en dedicar todo el tiempo de la clase en el uso exclusivo del software. Se recomienda solo usar este programa como complemento en el proceso de enseñanza-aprendizaje en Geometría.

La experiencia didáctica en fusionar la mediación del docente en la comprensión de los objetos geométricos, con la utilización de una herramienta tecnológica, como es el GeoGebra, evidencia la apropiación, por parte de los estudiantes, de conocimientos geométricos, tanto en coordenadas cartesianas de dos y tres dimensiones, como en coordenadas polares, además de una buena participación y motivación de los estudiantes en el trabajo de aula, individual y colaborativo, puesto que por el simple hecho de tener disponible una herramienta tecnológica como el GeoGebra en sus dispositivos móviles y laptops, entre otras, en las clases de Matemáticas, los estudiantes muestran buena disposición al abordar un problema, y en general la metodología de la clase se ve influenciada positivamente.

Con miras a ir perfeccionando la introducción del GeoGebra en el proceso de enseñanza- aprendizaje de la Geometría, para la buena administración del tiempo en el aula, se sugiere tener en cuenta los siguientes aspectos: focalizar las instrucciones del GeoGebra que se usarán en clases; que el docente medie los principales conceptos, propiedades y resultados de la Geometría; hacer una estructuración adecuada de las tareas para la utilización del software; y por último, la organización en un tiempo pertinente del trabajo en las clases.

Es importante que los futuros docentes de Matemáticas experimenten en su formación la articulación del conocimiento tecnológico con el conocimiento pedagógico y disciplinar, integrando los tres tipos de conocimientos, entendiendo que la tecnología no es un recurso mágico que soluciona los problemas de aprendizaje ni que debe ser el centro de la clase, sino que es una herramienta más en el proceso de enseñanza-aprendizaje.

\section{Referencias}

Barahona, F., Barrera, O., Vaca, B., \& Hidalgo, H. (2015). GeoGebra para la enseñanza de la Matemática y su incidencia en el rendimiento académico estudiantil. Revista Tecnológica ESPOL - RTE, 28(5), 121-132. https://bit.ly/2uaUsvl

Calvo, M. (2008). Enseñanza Eficaz de la Resolución de Problemas en Matemáticas. Revista Educación, 32(1), 123-138. https://bit.ly/2TDsjHY

Corella, F. (2012, 20 de noviembre). Secciones Cónicas - FIT [video]. YouTube. https://bit.ly/2s]kJRc 
Cotic, N. (2014). GeoGebra como puente para aprender Matemática. Congreso Iberoamericano de Ciencia, Tecnología, innovación y educación, 1(2), 124-130. https://bit.ly/2NFNs0i

GeoGebra (2019). Una introducción al desarrollo de GeoGebra. Consultado el 13 de enero de 2019. http://dev.geogebra.org

González, C. (2010). El aprendizaje y el conocimiento académico sobre la enseñanza como claves para mejorar la docencia universitaria. Calidad en la Educación, (33), 123-146.

https://doi.org/10.31619/caledu.n33.141

Jiménez, J., \& Jiménez, S. (2017). GeoGebra, una propuesta para innovar el proceso enseñanza-aprendizaje. Revista Electrónica sobre Tecnología, Educación y Sociedad, 4(7), 1-17. https://bit.ly/2G604wG

Luna, L. (2012, 9 de mayo). Aplicaciones de las cónicas en la física [video]. YouTube.

https://bit.ly/2v6kBMn

Morales-López, Y. (2019). Conocimientos que evidencian los futuros profesores cuando realizan una tarea que involucra geometría, enseñanza y uso de tecnologías. Acta Scientiae, 21(2), 75-92.

https://doi.org/10.17648/acta.scientiae.v21 iss2id5081
Muñoz, J., Federman, J., \& Munévar, R. (2002). Experiencias en investigación-acción-reflexión con educadores en proceso de formación en Colombia. Revista Electrónica de Investigación Educativa, 4(1), 1-15. https://bit.ly/3apzv0d

Organización de Estados Iberoamericanos (2010). Metas Educativas 2021. La Educación que queremos para la generación de los Bicentenarios.

https://bit.ly/365UmlX

Ramírez, C. (2015). Diseño de herramientas que fomentan el aprendizaje de matemáticas con ayuda de Mathematica 10. Elementos, 5(5), 65-78.

https://doi.org/10.15765/e.v5i5.613

Silva-Peña, I. (2012). Investigación-Acción Realizada por Docentes Chilenos como una vía para su Desarrollo Profesional: Caracterización de dos experiencias. Paradigma, 33(1), 27-44.

https://bit.ly/3675xek

Socas, M. (2011). La enseñanza del Álgebra en la Educación Obligatoria. Aportaciones de la investigación. Números, Revista de Didáctica de las Matemáticas, 77, 5-34. https://bit.ly/2NJxXod

Universidad Central de Chile (2017). Manual de Apoyo Docente. Evaluación para el aprendizaje. https://bit.ly/2NEfedx

\section{CÓMO CITAR:}

Rojas-Bello, R. R. (2020). Introducción del GeoGebra en el proceso de enseñanzaaprendizaje de Geometría a docentes en formación. Revista Caribeña de Investigación Educativa (RECIE), 4(1), 124-134. https://doi.org/10.32541/recie.2020. v4i1.pp124-134 